\title{
Unification, KK-thresholds and the top Yukawa coupling in F-theory GUTs
}

\author{
G.K. Leontaris ${ }^{(1)}$, N.D. Tracas ${ }^{(2)}$ and G. Tsamis ${ }^{(2)}$ \\ ${ }^{1}$ Theoretical Physics Division, Ioannina University, GR-45110 Ioannina, Greece \\ ${ }^{2}$ Physics Department, National Technical University, 15773 Athens, Greece
}

\begin{abstract}
In a class of F-theory $S U(5)$ GUTs the low energy chiral mass spectrum is obtained from rank one fermion mass textures with a hierarchical structure organised by $U(1)$ symmetries embedded in the exceptional $E_{8}$ group. In these theories chiral fields reside on matter 'curves' and the tree level masses are computed from integrals of overlapping wavefuctions of the particles at the triple intersection points. This calculation requires knowledge of the exact form of the wavefuctions. In this work we propose a way to obtain a reliable estimate of the various quantities which determine the strength of the Yukawa couplings. We use previous analysis of KK threshold effects to determine the (ratios of) heavy mass scales of the theory which are involved in the normalization of the wavefunctions. We consider similar effects from the chiral spectrum of these models and discuss possible constraints on the emerging matter content. In this approach, we find that the Yukawa couplings can be determined solely from the $U(1)$ charges of the states in the 'intersection' and the torsion which is a topological invariant quantity. We apply the results to a viable $S U(5)$ model with minimal spectrum which satisfies all the constraints imposed by our analysis. We use renormalization group analysis to estimate the top and bottom masses and find that they are in agreement with the experimental values.
\end{abstract}




\section{Introduction}

Recent progress in F-theory model building $[1-8]^{1}$ has shown that old successful GUTs, including the Georgi-Glashow minimal SU(5), the SO(10) model etc, are naturally realised on the world-volume of non-perturbative seven branes wrapping appropriate compact surfaces. The rather interesting fact in F-theory constructions is that they are defined on a compact elliptically fibered Calabi-Yau complex four dimensional manifold thus the exceptional groups $E_{6}, E_{7}, E_{8}$, can be naturally incorporated into the theory too [1 $[3,6]$. Although exceptional gauge symmetries suffer from several drawbacks when realized in the context of four-dimensional grand unified theories, in the case of F-theory models they are more promising as new possibilities arise for the symmetry breaking mechanisms and the derivation of the desired massless spectrum.

Present studies on F-theory model building have been concentrated on three generation -mainly $S U(5)$ - GUT models which fall into the following two distinct categories: those where all three families with the same Standard Model representation content are assigned to a single matter curve [11-14], and variants [15-21] where some or all of the quark and lepton families are assigned to different curves. Several of these constructions built up to these days have attempted to give solutions to fundamental GUT problems as is the case of doublet-triplet splitting, the rapid proton decay, the Higgs mixing term, the neutrino sector and other related issues [3, 6, 10,16,21, 23]. To analyse the phenomenological properties one should extract the relevant information from the superpotential which can be readily constructed once a particular assignment of the fermion families and Higgs on the matter curves has been chosen. Of course, dominant rôle on the estimation of such effects is played by the Yukawa couplings, thus the theory's predictive power depends on the calculability of the latter.

In F-theory GUTs the trilinear Yukawa couplings are realised at the intersections of three matter curves $\Sigma_{i}, i=1,2,3$ where the zero-modes of two fermion fields and a Higgs boson reside. Along these curves the $G_{S}$ symmetry is enhanced $G_{\Sigma_{i}} \supset G_{S} \times U(1)_{i}$ while the corresponding zero modes are charged under the $U(1)_{i}$. To determine the most general structure of the zero-mode wavefunctions one has to solve their corresponding differential equations of motion emerging from the twisted eight-dimensional YangMills action [2], (see also [14,24,26]). In general, the solutions are found to exhibit the expected gaussian [24,25] profile which falls off exponentially away from the curve while their exact form is specified by a mass scale characterizing the size of the compact space and the particular $U(1)_{i}$-charge of the relevant zero-mode. The Yukawa couplings of the $\{33\}$-entries of the up, down and charged lepton mass matrices are then computed in terms of the integrals of overlapping wavefunctions of the aforementioned form at the intersection point of three matter curves

$$
\lambda_{i j} \propto M_{*}^{4} \int_{S} \psi_{i} \psi_{j} \phi d z_{1} \wedge d \bar{z}_{1} \wedge d z_{2} \wedge d \bar{z}_{2}
$$

where the integration is over the compact internal four-dimensional manifold $S$ supporting the gauge group of the theory, $\psi, \phi$ denote the fermion and Higgs wavefunctions

\footnotetext{
${ }^{1}$ For other recent related work and a review see also [9, 10
} 
respectively whilst $M_{*}$ is associated to the supergravity limit of the compactified theory, hence it will be naturally linked to the F-theory compactification scale. Furthermore, assuming that higher order non-renormalizable Yukawa couplings are generated through mediation of heavy string modes and/or Kaluza-Klein states, the calculation of all Yukawa entries of this type in the fermion mass matrices can be reduced to a similar computation [18.

To quantify low energy implications of a given model, a reliable estimation of the integral (1) is required. This is however a non-trivial task since, although the local profile of the wavefunction is precisely known, the final result is expressed in terms of several parameters which mainly appear in the normalization of the wavefunction. In particular, the background scalar field vev, the $M_{G U T}$ scale, the scale $M_{*}$ as well as the geometry of the surface $S$ supporting the GUT symmetry are not precisely known. Estimates of such global quantities have been given and rather sensible results have been obtained in the case of the mass hierarchy [12, 15, 18. However, in this context one fails to predict a heavy top mass compatible with the experimental data in an unambiguous manner.

In this work we reconsider the problem of trilinear Yukawa couplings in F-theory GUTs, focussing on the normalization of the wavefunction and the rôle of the various scales of the theory. We rely on the results of [27] and [5] to determine the effects of KK-massive modes on the renormalization group running the GUT scale and the gauge unification. Using these results we estimate the modifications of mass scales ratio $M_{G U T} / M_{C}$ and express this solely in terms of the Ray-Singer torsion [28] which is a topologically invariant quantity. Identifying the various scales appearing in the computation of the overlapping integrals with $M_{G U T}$ and $M_{C}$, we argue that this gives a reliable computation for the top Yukawa coupling. The final result for the Yukawa coupling depends only on the charges of the fields involved and an exponential factor involving the torsion.

In the next section we review in brief the basic F-theory set up and the relevance of the Ray-Singer torsion to the threshold corrections. We show how KK-massive modes modify the GUT scale and in section 3 we perform a similar analysis for the case of chiral matter subject to flux constraints. In section 4 we apply the analysis in a realistic F-theory $S U(5)$ GUT [18] which was shown to reproduce successfully the fermion hierarchy and confront other main phenomenological issues. In sections 5 and 6 we perform the renormalization group analysis and obtain the formulae for the top and bottom Yukawa couplings by computing the corresponding integrals. As already said the result is expressed in terms of the analytic torsion. As an application, we perform an explicit numerical calculation of the top and bottom Yukawa couplings choosing a specific line bundle on a Hirzebruch surface. In section 7 we summarize our work and present our conclusion. Some technical details regarding the solutions of the renormalization group equations for the computation of the third generation quark masses are shown in the appendix. 


\section{KK-modes and the GUT scale}

Our F-theory set up is assumed to be the world-volume of the seven-brane of a ADEtype singularity which wraps the space $R^{3,1} \times S$ where $S$ is a Kähler manifold of two complex dimensions. At low energies, F-theory is described by an eight-dimensional Yang-Mills theory on $R^{3,1} \times S$ which must be topologically twisted to preserve $N=1$ supersymmetry [2, 3]. The supersymmetric multiplet consists of the gauge field, two scalars $\Phi_{8,9}$ combined into the complex fields $\phi / \bar{\phi}=\Phi_{8} \pm i \Phi_{9}$ and the fermions $\eta, \psi, \chi$ all in the adjoint of the gauge group. In the twisted theory $\eta, \psi, \chi$ appear as holomorphic $(k, 0)$ forms (and their complex conjugate antiholomorphic $(0, l))$ with $k, l=0,1,2$, while the scalar $\phi$ is a two form. At the $d=4, N=1$ level, these fields are organised as one gauge and two chiral multiplets

$$
\left(A_{\mu}, \eta\right),\left(A_{\bar{m}}, \psi_{\bar{m}}\right),\left(\phi_{12}, \chi_{12}\right)
$$

For the computation of threshold corrections we assume that the gauge theory model is described by some GUT gauge group which for definiteness we take it to be $G_{S}=S U(5)$ (at a scale $M_{G U T} \ll M_{\text {Planck }}$ ). In this limit it is also natural to assume that KK-modes are much lighter than other string excitations.

Threshold corrections are expressed in terms of the masses of the KK-spectrum which emerge as the non-zero eigenvalues of the Laplacian of the eight dimensional theory. Therefore, threshold corrections are constants and should be independent of the particular metric on the surface $S$, while they are expected to be expressed in terms of a topologically invariant quantity. It was first shown, already sometime ago [27], that in the context of $M$-theory compactified on a manifold with $G_{2}$ holonomy the most appropriate topological invariant quantity to express these threshold corrections is the Ray-Singer torsion [28]. A similar analysis has been performed for the case of $F$-theory in [5] which we now review in brief.

The $S U(5)$ subgroup which commutes with the Standard Model $\left(G_{S M}\right)$ is the $U(1)_{Y}$. We denote with $q_{i}$ the $U(1)_{Y}$ charge of the Standard Model representations $R_{i}$ arising from the decomposition of the adjoint of $S U(5)$ under $G_{S M}$

$$
24 \rightarrow(8,1)_{0}+(1,3)_{0}+(1,1)_{0}+(3,2)_{-5 / 6}+(\overline{3}, 2)_{5 / 6}
$$

We assume that the GUT group breaks to $G_{S M}$ by turning on a flux along $U(1)_{Y}$, hence each $q_{i}$ determines now a line bundle which we denote it by $\mathcal{L}^{q_{i}}$. We observe that in the $S U(5)$ adjoint decomposition (2), in addition to the standard $S U(3), S U(2)$ adjoints there are in principle exotic zero modes in the $(3,2)$ and $(\overline{3}, 2)$ representations along the $\mathcal{L}^{ \pm 5 / 6}$ line bundles whose numbers are given by the Euler character $-\chi\left(S, \mathcal{L}^{ \pm 5 / 6}\right)$. Therefore, elimination of these massless modes requires $\chi\left(S, \mathcal{L}^{ \pm 5 / 6}\right)=0$ which implies 2 the following relation for the cohomology class: $c_{1}\left(\mathcal{L}^{5 / 6}\right) \cdot c_{1}\left(\mathcal{L}^{5 / 6}\right)=-2$.

Consider next the Dolbault operator $\bar{\partial}$ of the corresponding holomorphic bundle $V$ with representation $R(V)$, acting on $k$-forms as follows

$$
\bar{\partial}: \Omega_{S}^{0, k} \otimes R(V) \rightarrow \Omega_{S}^{0, k+1} \otimes R(V), \text { for } k=0,1
$$

\footnotetext{
${ }^{2}$ The number of massless modes of a $\mathcal{T}_{i}$ representation is $-\chi\left(S, \mathcal{T}_{i}\right)=1+\frac{1}{2} c_{1}\left(\mathcal{T}_{i}\right) \cdot\left(c_{1}\left(\mathcal{T}_{i}\right)+c_{1}(S)\right)$ while here the following relation is satisfied: $c_{1}\left(\mathcal{L}^{5 / 6}\right) \cdot c_{1}(S)=0$ ].
} 
and the Laplacian

$$
\Delta_{k, R(V)}=\left(\bar{\partial}+\bar{\partial}^{\dagger}\right)^{2}=\bar{\partial} \bar{\partial}^{\dagger}+\bar{\partial}^{\dagger} \bar{\partial}
$$

If we denote collectively with $\psi_{k}^{n}$ its $k$-form eigenfunction then

$$
\Delta_{k, R(V)} \psi_{k}^{n}=\lambda_{n}^{k} \psi_{k}^{n}
$$

where $\lambda_{n}^{k}$ represents the corresponding eigenvalue and in four dimensions corresponds to a squared mass.

We express now the threshold corrections of the gauge multiplet in terms of the Ray-Singer torsion. The running of the gauge couplings are governed by the equation

$$
\frac{16 \pi^{2}}{g_{a}^{2}(\mu)}=\frac{16 \pi^{2} k_{a}}{g_{s}^{2}}+b_{a} \log \frac{\Lambda^{2}}{\mu^{2}}+\mathcal{S}_{a}^{(g)}, \quad a=3,2, Y
$$

where $\Lambda$ is the gauge theory cutoff scale, $k_{a}=(1,1,5 / 3)$ are the normalization coefficients for the usual embedding of the Standard Model to $S U(5), g_{s}$ is the gauge coupling as deduced from the higher theory and $b_{a}$ are the one-loop $\beta$-function coefficients which are given by 3

$$
b_{a}=2 \operatorname{Str}_{M=0} Q_{a}^{2}\left(\frac{1}{12}-\chi^{2}\right)
$$

where $\chi$ is the helicity operator, Str denotes the supertrace (bosons contribute with weight +1 and fermions with -1 ) and $Q_{a}$ stands for the three generators of the Standard Model gauge group $S U(3) \times S U(2) \times U(1)_{Y}$. Finally, $\mathcal{S}_{a}^{(g)}$ correspond to the one-loop threshold corrections and are given by a similar term over the massive states (in contrast with the previous term that corresponds to massless states)

$$
\begin{aligned}
S_{a}^{(g)} & =2 \sum_{R_{i}} \operatorname{Str}_{M \neq 0} Q_{a}^{2}\left(\frac{1}{12}-\chi^{2}\right) \log \left(\Lambda^{2} / M^{2}\right) \\
& =2 \sum_{i} \operatorname{Tr}_{R_{i}} Q_{a}^{2} \operatorname{Str}_{M \neq 0}\left(\frac{1}{12}-\chi^{2}\right) \log \left(\Lambda^{2} / M^{2}\right)
\end{aligned}
$$

In the second line $\operatorname{Str}_{M \neq 0} Q_{a}^{2}$ has been factored out since it depends only on the representation $R_{i}$ (we are suppressing the notation for the bundle $V$ in $R_{i}(V)$ ). For a certain helicity state there is a logarithmic dependence on its mass squared $M^{2}$ which corresponds to the eigenvalue of the Laplacian $\Delta_{k, R_{i}}$. Further, since the trace of log is equal to the logarithm of the determinant we can replace the trace of $\log \Lambda^{2} / M^{2}$ above with

$$
-\log \operatorname{det}^{\prime}\left(\Delta_{k, R_{i}} / \Lambda^{2}\right)
$$

where the prime on det denotes that we exclude the zero modes.

Now, each eigenvector of the zero-form Laplacian $\Delta_{0, R_{i}}$ contributes a vector multiplet with helicities $1,-1, \frac{1}{2},-\frac{1}{2}$, while the one-form Laplacian $\Delta_{1, R_{i}}$ gives a chiral

\footnotetext{
${ }^{3}$ We adopt here the notation of [27].
} 
multiplet with helicities $0,0, \frac{1}{2},-\frac{1}{2}$. Similarly, $\Delta_{2, R_{i}}$ is associated to anti-chiral multiplets. Evaluating the supertrace we get

$$
\operatorname{Str}\left(\frac{1}{12}-\chi^{2}\right)= \begin{cases}-\frac{3}{2} & \text { for the vectot multiplet } \\ +\frac{1}{2} & \text { for the chiral multiplet }\end{cases}
$$

Therefore, the total sum of the contribution of KK-modes to the thresholds from the gauge fields is then written

$$
\mathcal{S}_{a}^{(g)}=2 \sum_{i} \operatorname{Tr}_{R_{i}}\left(Q_{a}^{2}\right) \mathcal{K}_{i}
$$

with

$$
\mathcal{K}_{i}=\frac{3}{2} \log \operatorname{det}^{\prime} \frac{\Delta_{0, R_{i}}}{\Lambda^{2}}-\frac{1}{2} \log \operatorname{det}^{\prime} \frac{\Delta_{1, R_{i}}}{\Lambda^{2}}-\frac{1}{2} \log \operatorname{det}^{\prime} \frac{\Delta_{2, R_{i}}}{\Lambda^{2}}
$$

and

$$
\operatorname{Tr}_{R_{0}}\left(Q_{\{3,2, Y\}}^{2}\right)=\left\{\begin{array}{lll}
\{3,2,0\}, & \text { for } & (\mathbf{8}, \mathbf{1})_{\mathbf{0}}+(\mathbf{1}, \mathbf{3})_{\mathbf{0}}+(\mathbf{1}, \mathbf{1})_{\mathbf{0}} \\
\left\{1, \frac{3}{2}, \frac{25}{6}\right\}, & \text { for } \quad(\mathbf{3}, \mathbf{2})_{-\mathbf{5} / \mathbf{6}},(\overline{\mathbf{3}}, \mathbf{2})_{\mathbf{5} / \mathbf{6}}
\end{array}\right.
$$

with normalization $\operatorname{Tr}\left(Q_{a}^{2}\right)=\frac{k_{a}}{2}$. Therefore, the three $\mathcal{S}_{a}^{(g)}$ read

$$
\begin{aligned}
\mathcal{S}_{Y}^{(g)} & =\frac{25}{3}\left(\mathcal{K}_{5 / 6}+\mathcal{K}_{-5 / 6}\right) \\
\mathcal{S}_{2}^{(g)} & =4 \mathcal{K}_{0}+3\left(K_{5 / 6}+\mathcal{K}_{-5 / 6}\right) \\
\mathcal{S}_{3}^{(g)} & =6 \mathcal{K}_{0}+2\left(\mathcal{K}_{5 / 6}+\mathcal{K}_{-5 / 6}\right)
\end{aligned}
$$

with an obvious notation in the subscript of $\mathcal{K}$. Further, the spectrum of $\Delta_{1}$ is equivalent to the sum of the spectra of $\Delta_{0}$ and $\Delta_{2}$. Then we can write (13) as follows

$$
\mathcal{K}_{i}=2 \log \operatorname{det}^{\prime} \frac{\Delta_{0, R_{i}}}{\Lambda^{2}}-\log \operatorname{det}^{\prime} \frac{\Delta_{1, R_{i}}}{\Lambda^{2}}
$$

Introducing the Ray-Singer torsion

$$
\mathcal{T}_{R}=\frac{1}{2} \sum_{k=0}^{2}(-1)^{k+1} \log \operatorname{det}^{\prime} \frac{\Delta_{k, R_{i}}}{\Lambda^{2}}
$$

and noting that the Laplacian commutes with the Hodge $*$ operator which maps $k$-forms to $(2-k)$-forms, so that $\Delta_{k, R}$ and $\Delta_{2-k, R^{*}}$ have the same spectrum, we get 5 ]

$$
\mathcal{T}_{R}=-\mathcal{T}_{R^{*}}=\frac{1}{2}\left(2 \log \operatorname{det}^{\prime} \frac{\Delta_{0, R_{i}}}{\Lambda^{2}}-\log \operatorname{det}^{\prime} \frac{\Delta_{1, R_{i}}}{\Lambda^{2}}\right)
$$

The expression inside the parenthesis is exactly what we have derived previously for the threshold corrections. According to the Ray and Singer theorem [28] the torsion is 
independent of the metric of the manifold and therefore independent of the cutoff scale $\Lambda$. (The latter can be eliminated by scaling the metric.)

In the above expression for the torsion it has been assumed that there are no zero modes as is the case of a non-trivial representation $R_{i}$. However, in the case of the trivial bundle there are zero modes and the torsion is not equal to $\mathcal{K}_{R_{0}}$. These are related by

$$
2 \mathcal{T}_{\mathcal{O}}=\mathcal{K}_{\mathcal{O}}-\log \left(V \Lambda^{2}\right)
$$

where in the subscript we used the standard notation $\mathcal{O}$ for the trivial representation.

We assume $S$ to be a del Pezzo surface and we expect that $\mathcal{K}_{5 / 6}=\mathcal{K}_{-5 / 6}$ so that the equations in (15) can be cast in the form

$$
\left(\mathcal{S}_{Y}^{(g)}, \mathcal{S}_{2}^{(g)}, \mathcal{S}_{3}^{(g)}\right)=\left(\frac{50}{3} \mathcal{K}_{5 / 6}, 6 \mathcal{K}_{5 / 6}+4 \mathcal{K}_{0}, 4 \mathcal{K}_{5 / 6}+6 \mathcal{K}_{0}\right)
$$

Taking into account that the contribution of the gauge multiplet to the $\beta$-function is $b_{a}^{(g)}=(0,-6,-9)$ we can rewrite the above as

$$
\mathcal{S}_{a}^{(g)}=\frac{2}{3} b_{a}^{(g)}\left(\mathcal{K}_{5 / 6}-\mathcal{K}_{0}\right)+10 k_{a} \mathcal{K}_{5 / 6}=\frac{4}{3} b_{a}^{(g)}\left(\mathcal{T}_{5 / 6}-\mathcal{T}_{0}\right)+20 k_{a} \mathcal{T}_{5 / 6}
$$

The second term is proportional to $k_{a}$ and therefore it can be absorbed to the $16 \pi^{2} k_{a} / g_{s}^{2}$ term, redefining the coupling $g_{s}$. And, finally, the initial equation (6) for the running of the gauge coupling, where only the gauge multiplet has been taken into account, gives

$$
\begin{aligned}
\frac{16 \pi^{2}}{g_{a}^{2}(\mu)} & =\frac{16 \pi^{2} k_{a}}{g_{s}^{2}}+b_{a}^{(g)} \log \frac{\Lambda^{2}}{\mu^{2}}+\mathcal{S}_{a}^{(g)} \\
& =\frac{16 \pi^{2} k_{a}}{g_{s}^{2}}+b_{a}^{(g)} \log \frac{\exp \left[4 / 3\left(\mathcal{T}_{5 / 6}-\mathcal{T}_{0}\right)\right]}{\mu^{2} V^{1 / 2}}
\end{aligned}
$$

where all the $\Lambda$ dependence, after the appropriate cancellation between the massless and massive modes, has been absorbed in the redefinition of the bare gauge coupling along with the second term of (21), and we can define the $M_{G U T}$ as

$$
M_{G U T}^{2}=\frac{\exp \left[4 / 3\left(\mathcal{T}_{5 / 6}-\mathcal{T}_{0}\right)\right]}{V^{1 / 2}}
$$

If we associate the world volume factor $V^{-1 / 4}$ with the characteristic compactification scale $M_{C}$, we can write this equation as follows

$$
M_{G U T}=e^{2 / 3\left(\mathcal{T}_{5 / 6}-\mathcal{T}_{0}\right)} M_{C} .
$$

Thereby, we conclude that the ratio $M_{G U T} / M_{C}$ of the two characteristic scales of the theory depends only on the torsion and is topologically invariant. 


\section{The inclusion of chiral matter}

We have seen in the previous section that the contribution of threshold corrections from KK-modes of the vector multiplet leads to a cutoff independent RG running of the gauge couplings. We wish now to include the analogous contributions from the chiral and Higgs sector of the theory. In F-theory constructions we need to take into account the zero mode as well as the KK-massive mode contributions.

We first start by incorporating the zero mode effects. In the case of the $S U(5)$ theory we discuss in this paper the possible non-trivial representations transform as $10, \overline{10}$ and $5, \overline{5}$. Matter fields in general, will contribute to the RG running in (22) with a term of the form $b_{a}^{x} \log \frac{\Lambda^{\prime 2}}{\mu^{2}}$ where $b_{a}^{x}$ are the $\beta$-function coefficients for the corresponding three SM gauge group factors and $\Lambda^{\prime}$ a cutoff scale not necessarily equal to the gauge cutoff $\Lambda$. If matter fields in the theory arise in complete $S U(5)$ multiplets, then the $\beta$-functions contribute in proportion to the coefficients $k_{a}$, ie. $b_{a}^{x} \propto k_{a}$. Then writing $\log \frac{\Lambda^{\prime 2}}{\mu^{2}}$ as $\log \frac{\Lambda^{\prime 2}}{M_{G U T}^{2}}+\log \frac{M_{G U T}^{2}}{\mu^{2}}$ we can absorb the first logarithm in a redefinition of the gauge coupling

$$
\frac{16 \pi^{2}}{g_{G U T}^{2}} k_{a} \equiv \frac{4 \pi}{a_{G U T}} k_{a}=\frac{4 \pi}{a_{S}} k_{a}+b_{a}^{x} \log \frac{\Lambda^{\prime 2}}{M_{G U T}^{2}}
$$

The massless chiral and Higgs spectrum at low energies, however, does not form complete $S U(5)$ multiplets. As it is well known, Higgs doublets are found in $5+\overline{5}$ representations of $S U(5)$ together with the dangerous color triplets. To protect proton from rapid decay, triplets must receive a mass at a relatively high scale $M_{X} \leq M_{G U T}$. Taking this into account we write $b_{a}^{x}=b_{a}+b_{a}^{T}$ where $b_{a}$ denotes the MSSM $\beta$-functions while $b_{a}^{T}$ the color triplet part. Adding all contributions, (22) takes the form

$$
\frac{16 \pi^{2}}{g_{a}^{2}(\mu)}=k_{a} \frac{16 \pi^{2}}{g_{G U T}^{2}}+\left(b_{a}^{(g)}+b_{a}\right) \log \frac{M_{G U T}^{2}}{\mu^{2}}+b_{a}^{T} \log \frac{M_{G U T}^{2}}{M_{X}^{2}}
$$

It should be mentioned that if the contributions from matter fields fail to obey the condition $b_{a}^{x} \propto k_{a}$, then (25) splits the common gauge coupling to three different values at $M_{G U T}$.

We discuss now the threshold contributions arising from the $\Sigma_{\overline{5}}$ and $\Sigma_{10}$ matter curves. From the decompositions

$$
10 \rightarrow(3,2)_{\frac{1}{6}}+(\overline{3}, 1)_{-\frac{2}{3}}+(\mathbf{1}, \mathbf{1})_{1}, \quad \overline{\mathbf{5}} \rightarrow(\overline{\mathbf{3}}, \mathbf{1})_{\frac{1}{3}}+(\mathbf{1}, \mathbf{2})_{-\frac{1}{2}}
$$

we readily find that KK-modes residing on these curves contribute to thresholds as follows [5]

$$
\begin{aligned}
S_{a}^{\overline{5}} & =\left(\mathcal{K}_{1 / 3}, \mathcal{K}_{-1 / 2}, \mathcal{K}_{-1 / 2}+2 / 3 \mathcal{K}_{1 / 3}\right) \\
S_{a}^{10} & =\left(2 \mathcal{K}_{1 / 6}+\mathcal{K}_{-2 / 3}, 3 \mathcal{K}_{1 / 6}, 1 / 3 \mathcal{K}_{1 / 6}+2 \mathcal{K}_{1}+8 / 3 \mathcal{K}_{-2 / 3}\right)
\end{aligned}
$$

\footnotetext{
${ }^{4}$ In the next sections we will see however that gauge coupling splitting at $M_{G U T}$ is not avoided when a non-trivial $U(1)_{Y}$ flux is turned on to break $S U(5)$.
} 
where, as previously, $\mathcal{K}_{i}$ stand for the Ray-Singer torsion. As in the case of gauge fields one can absorb the parts proportional to $k_{a}$ by redefinition of the initial gauge coupling. Further, it is anticipated that on appropriate bundle structures such corrections will diminish or be negligible compared to other (from light degrees of freedom or supersymmetry) threshold effects [29]- [32].

In F-theory constructions one of the possible ways to break the GUT symmetries is to turn on a flux on the worldvolume of the seven-brane supporting the unified gauge group. In the present case, the $S U(5)$ gauge symmetry can be broken by turning on a non-trivial flux along the hypercharge with $Q_{Y}=\operatorname{diag}\left\{-\frac{1}{3},-\frac{1}{3},-\frac{1}{3}, \frac{1}{2}, \frac{1}{2}\right\}$. As a result, $S U(5)$ multiplets residing on certain curves where the flux restricts non-trivially, might split. In the case of Higgs fiveplets in particular, this mechanism could be used to eliminate the unwanted triplets from the light spectrum, however the effective theory may no longer contain complete $S U(5)$ representations and the proportionality conditions $b_{a}^{x} \propto k_{a}$ may not hold. We analyse this issue in the remaining of this section.

Suppose that $M_{10}, M_{5}$ are two integers representing the number of complete SU(5) 10 and $\overline{5}$ representations in a specific construction. We work in the context of spectral cover approach, thus we consider the $E_{8}$ embedding of $S U(5)_{G U T}$ and the breaking

$$
E_{8} \rightarrow S U(5)_{G U T} \times U(1)^{4}
$$

The $S U(5)$ chiral and Higgs matter fields descend from the adjoint representation of the $E_{8}$ symmetry and reside on the various curves denoted with $\Sigma_{10_{j}}, \Sigma_{\overline{5}_{i}}$. We expect that the $U(1)$ fluxes (those not included in $S U(5)_{G U T}$ ) together with the tracelessness condition $\sum_{i} F_{U(1)_{i}}=0$ imply the following condition on the numbers of multiplets [17]

$$
\sum_{i} M_{5}^{i}+\sum_{j} M_{10}^{j}=0
$$

Consider first the case that we have all 10 type chiral matter accommodated only on one $\Sigma_{10}$ curve and all chiral states $\overline{5}$ respectively on a single $\Sigma_{\overline{5}}$ curve. Then condition (28) implies the relation $M_{10}=-M_{5}=M$.

We denote with $N_{Y_{5}}, N_{Y_{10}}$ the corresponding units of $Y$ flux which splits the $S U(5)$ multiplets according to

$$
\Sigma_{\overline{5}}:\left\{\begin{array}{l}
n_{(\mathbf{3}, \mathbf{1})_{-1 / 3}}-n_{(\overline{\mathbf{3}}, \mathbf{1})_{1 / 3}}=M_{5} \\
n_{(\mathbf{1}, \mathbf{2})_{1 / 2}}-n_{(\mathbf{1}, \mathbf{2})_{-1 / 2}}=M_{5}+N_{Y_{5}}
\end{array} \quad \Sigma_{10}:\left\{\begin{array}{l}
n_{(\mathbf{3}, \mathbf{2})_{1 / 6}}-n_{(\overline{\mathbf{3}}, \mathbf{2})_{-1 / 6}}=M_{10} \\
n_{(\overline{\mathbf{3}}, \mathbf{1})_{-2 / 3}}-n_{(\mathbf{3}, \mathbf{1})_{2 / 3}}=M_{10}-N_{Y_{10}} \\
n_{(\mathbf{1}, \mathbf{1})_{1}}-n_{(\mathbf{1}, \mathbf{1})_{-1}}=M_{10}+N_{Y_{10}}
\end{array}\right.\right.
$$

Notice that these formulae count the number of 5 -components minus those of $\overline{5}$ and the number of 10 components minus those of $\overline{10}$. Since we know that families are accommodated on $\overline{5}$ 's we require $n_{(\overline{\mathbf{3}}, \mathbf{1})_{1 / 3}}>n_{(\mathbf{3}, \mathbf{1})_{-1 / 3}}$ which implies $M_{5}<0$. Similarly, because the remaining pieces of fermion generations live on 10's, we wish to end up with 10-components after the symmetry breaking, hence we should have $M_{10}>0$. For example, for exactly three generations we should demand $M_{10}=-M_{5}=3$ and $N_{Y_{j}}=0$. 
In general however, various curves belong to different homology classes and flux restricts non-trivially to some of them, thus $N_{Y_{j}} \neq 0$ at least for some values of $j$. Furthermore, we note that in the above description we deal with negative numbers which are used to discriminate the $\overline{5}, \overline{10}$ conjugate representations from 5 's and 10 's. In order to take correctly into account their contributions to the $\beta$-functions, care should be taken so that only positive numbers are introduced. Thus for the number of triplets we take

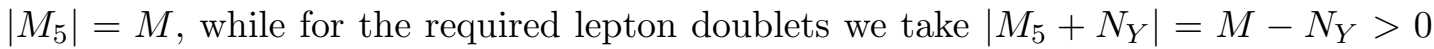
and so on 5 . For this simple example we observe that the contributions to $\beta$-functions come with the right proportionality factors $1: 1: \frac{5}{3}=k_{3}: k_{2}: k_{1}$ only in the unrealistic case $N_{Y_{5}}=N_{Y_{10}}=N$ where $\frac{3}{5} b_{Y}=b_{3}=b_{2}=2 M-\frac{1}{2} N$, so that the arbitrary cutoff $\Lambda^{\prime}$ can be removed to a redefinition of the gauge coupling as in the case of the gauge fields.

In the general case the situation is more complicated. In the $E_{8}$ embedded $S U(5)$ case in particular, in principle there exist ten $\Sigma_{5}$ and five $\Sigma_{10}$ curves 6 where the corresponding $S U(5)$ representations may reside. The implementation of monodromy actions may reduce the number of these curves, however, the construction of realistic effective GUT theories capable of reproducing the know hierarchical spectrum eventually require the involvement of matter fields arising from a variety of matter curves. Furthermore, because of $U(1)_{Y}$-flux effects several $S U(5)$ representations split in a phenomenologically prescribed (and sometimes promiscuous) way resulting into incomplete multiplets.

Bearing in mind the above general context, we write the contribution to the $\beta$ functions as follows

$$
\begin{aligned}
b_{3}= & \frac{1}{2} \sum_{i}\left|M_{5}^{i}\right|+\sum_{j}\left|M_{10}^{j}\right|+\frac{1}{2} \sum_{j}\left|M_{10}^{j}-N_{10 Y}^{j}\right| \\
b_{2}= & \frac{1}{2} \sum_{i}\left|M_{5}^{i}+N_{5 Y}^{i}\right|+\frac{3}{2} \sum_{j}\left|M_{10}^{j}\right| \\
b_{Y}= & \frac{1}{2} \sum_{i}\left|M_{5}^{i}+N_{5 Y}^{i}\right|+\frac{1}{3} \sum_{i}\left|M_{5}^{i}\right| \\
& +\frac{1}{6} \sum_{j}\left|M_{10}^{j}\right|+\frac{4}{3} \sum_{j}\left|M_{10}^{j}-N_{10 Y}^{j}\right|+\sum_{j}\left|M_{10}^{j}+N_{10 Y}^{j}\right|
\end{aligned}
$$

where $i, j$ summations are over the number of $\Sigma_{\overline{5}}$ and $\Sigma_{10}$ discrete curves in the quotient theory, i.e. after the monodromy action.

In order to acquire again the relation $(1,1,5 / 3)$ we first must impose the equality

\footnotetext{
${ }^{5}$ In fact here we assume that $\left|M_{5}\right|$ counts exactly the number of $(\overline{\mathbf{3}}, \mathbf{1})_{1 / 3}$ and that there are no extra $(\mathbf{3}, \mathbf{1})_{-1 / 3},(\overline{\mathbf{3}}, \mathbf{1})_{1 / 3}$ pairs and similarly for the other components, otherwise threshold effects should be taken into account [30,31,33. More general cases are considered in the next sections.

${ }^{6}$ This is clear from the decomposition of the adjoint of $E_{8}$, under $S U(5) \times S U(5)_{\perp}$

$$
248 \rightarrow(24,1)+(1,24)+(10,5)+(\overline{5}, 10)+(5, \overline{10})+(\overline{10}, \overline{5})
$$
}


between the contribution to $S U(3)$ and $S U(2)$

$$
\frac{1}{2} \sum_{i}\left|M_{5}^{i}\right|+\sum_{j}\left|M_{10}^{j}\right|+\frac{1}{2} \sum_{j}\left|M_{10}^{j}-N_{10 Y}^{j}\right|=\frac{1}{2} \sum_{i}\left|M_{5}^{i}+N_{5 Y}^{i}\right|+\frac{3}{2} \sum_{j}\left|M_{10}^{j}\right| \equiv \mathcal{B}
$$

where the parameter $\mathcal{B}$ was introduced for later convenience. We may rewrite the constraint above in a simplified way as follows

$$
\sum_{i}\left|M_{5}^{i}\right|-\sum_{i}\left|M_{5}^{i}+N_{5 Y}^{i}\right|=\sum_{j}\left|M_{10}^{j}\right|-\sum_{j}\left|M_{10}^{j}-N_{10 Y}^{j}\right|
$$

Now, we can reexpress the $U(1)_{Y}$ contribution in terms of $\mathcal{B}$ and a remaining quantity as follows

$$
b_{Y}=\frac{5}{3} \mathcal{B}-2 \sum_{j}\left|M_{10}^{j}\right|+\sum_{j}\left|M_{10}^{j}+N_{10 Y}^{j}\right|+\sum_{j}\left|M_{10}^{j}-N_{10 Y}^{j}\right|
$$

In (33) we have split the $U(1) \beta$-function into two parts, the first being $\frac{5}{3} \mathcal{B}$ which comparing with (31) we observe that preserves the required $k_{a}$-proportion with $b_{2,3}$. Therefore, the remaining part must be zero and finally we get the following two constraints

$$
\begin{aligned}
\sum_{j}\left|M_{10}^{j}\right|-\sum_{i}\left|M_{5}^{i}\right| & =\sum_{j}\left|M_{10}^{j}-N_{10 Y}^{j}\right|-\sum_{i}\left|M_{5}^{i}+N_{5 Y}^{i}\right| \\
2 \sum_{j}\left|M_{10}^{j}\right| & =\sum_{j}\left|M_{10}^{j}+N_{10 Y}^{j}\right|+\sum_{j}\left|M_{10}^{j}-N_{10 Y}^{j}\right|
\end{aligned}
$$

In writing the above contributions to the $\beta$-functions we have assumed that they correspond to all the matter content, including the 3 generations of the SM. Clearly, it is a non-trivial task to obtain a low energy effective field theory model with only the MSSM spectrum.

We note in passing that if we want to consider only the extra matter content, we should subtract a 3 from all absolute values: $\left|M_{5}^{i}\right| \rightarrow\left|M_{5}^{i}\right|-3,\left|M_{5}^{i}+N_{5 Y}^{i}\right| \rightarrow$ $\left|M_{5}^{i}+N_{5 Y}^{i}\right|-3$, etc. But since we know that the SM content forms a complete $\overline{5}$ and a 10 , the contributions from the 3 generations obey the rule $(1,1,5 / 3)$. Therefore, the above constraints hold also for the case when only the extra matter is considered. Note also that if all $N_{Y_{i}}$ are zero the relations hold automatically, as they should. Of course, any choice of $M$ 's has to satisfy the constraint (28).

\section{Application to a realistic minimal model}

From the analysis of the chiral and Higgs matter contributions to the $\beta$-functions presented above, it is clear that the cutoff independence constraints cannot be satisfied for any spectrum, even if flux and other consistency conditions are met. In this section we are going to discuss a realistic model with the minimal spectrum where these requirements are fulfilled. We make an explicit calculation to determine the gauge unification 


\begin{tabular}{|l|c|c|c|c|}
\hline Field & $U(1)_{i}$ & homology & $U(1)_{Y}$-flux & $U(1)$-flux \\
\hline $10^{(1)}=10_{3}$ & $t_{1,2}$ & $\eta-2 c_{1}-\chi$ & $-N$ & $M_{10_{1}}$ \\
\hline $10^{(2)}=10_{1}$ & $t_{3}$ & $-c_{1}+\chi_{7}$ & $N_{7}$ & $M_{10_{2}}$ \\
\hline $10^{(3)}=10_{2}$ & $t_{4}$ & $-c_{1}+\chi_{8}$ & $N_{8}$ & $M_{10_{3}}$ \\
\hline $10^{(4)}=10_{2}^{\prime}$ & $t_{5}$ & $-c_{1}+\chi_{9}$ & $N_{9}$ & $M_{10_{4}}$ \\
\hline $5^{(0)}=5_{h_{u}}$ & $-t_{1}-t_{2}$ & $-c_{1}+\chi$ & $N$ & $M_{5_{h_{u}}}$ \\
\hline $5^{(1)}=5_{2}$ & $-t_{1,2}-t_{3}$ & $\eta-2 c_{1}-\chi$ & $-N$ & $M_{5_{1}}$ \\
\hline $5^{(2)}=5_{3}$ & $-t_{1,2}-t_{4}$ & $\eta-2 c_{1}-\chi$ & $-N$ & $M_{5_{2}}$ \\
\hline $5^{(3)}=5_{x}$ & $-t_{1,2}-t_{5}$ & $\eta-2 c_{1}-\chi$ & $-N$ & $M_{5_{3}}$ \\
\hline $5^{(4)}=5_{1}$ & $-t_{3}-t_{4}$ & $-c_{1}+\chi-\chi_{9}$ & $N-N_{9}$ & $M_{5_{4}}$ \\
\hline $5^{(5)}=5_{h_{d}}$ & $-t_{3}-t_{5}$ & $-c_{1}+\chi-\chi_{8}$ & $N-N_{8}$ & $M_{5_{h_{d}}}$ \\
\hline $5^{(6)}=5_{y}$ & $-t_{4}-t_{5}$ & $-c_{1}+\chi-\chi_{7}$ & $N-N_{7}$ & $M_{5_{6}}$ \\
\hline
\end{tabular}

Table 1: Field representation content under $S U(5) \times U(1)_{t_{i}}$, their homology class and flux restrictions [17] for the model [18]. Superscripts in the first column are numbering the curves, while subscripts indicate the family, the Higgs etc. For convenience, only the properties of 10,5 are shown. $\overline{10}, \overline{5}$ are characterized by opposite values of $t_{i} \rightarrow-t_{i}$ etc. Note that the fluxes satisfy $N=N_{7}+N_{8}+N_{9}$ and $\sum_{i} M_{10_{i}}+\sum_{j} M_{5_{j}}=0$ while $\chi=\chi_{7}+\chi_{8}+\chi_{9}$.

and $b, t$-Yukawa couplings through the renormalization group running in a model which fulfills all the requirements.

To start with, we recall here the basic features of the model [18] which emerges from the general class [17] presented in Table 1. The first two columns give the field content under $S U(5) \times U(1)_{t_{i}}$ for the case of $Z_{2}$ monodromy. The third column presents the homology classes where $c_{1}$ is the first Chern class of the tangent bundle of GUT surface $S_{G U T}$ and $\eta=6 c_{1}-t$ with $-t$ being the first Chern class of the normal bundle to $S_{G U T}$. The $\chi_{i}$ are unspecified subject only to the condition $\chi=\chi_{7}+\chi_{8}+\chi_{9}$. If $\mathcal{F}_{Y}$ denotes the $U(1)_{Y}$ flux, to avoid a Green-Schwarz mass for the corresponding gauge boson we must require $\mathcal{F}_{Y} \cdot \eta=\mathcal{F}_{Y} \cdot c_{1}=0$. Then, we get $N_{i}=\mathcal{F}_{Y} \cdot \chi_{i}$ and consequently $N=\mathcal{F}_{Y} \cdot \chi=N_{7}+N_{8}+N_{9}$. Using these facts, all remaining entries of column 4 in Table 1 are easily deduced.

We now take the flux parameters to be $M_{10_{1,2,3}}=1, M_{5_{1,2,4}}=-1$ and $N=0$, while we have the freedom to choose $N_{7,8,9}$ subject only to the constraint $N=N_{7}+N_{8}+N_{9}$. This choice of $M_{i}, N_{j}$ 's ensures the existence of three 10 and three $\overline{5}$ representations which are needed to accommodate the three chiral families.

Next we use the $U(1)_{Y}$ flux mechanism to realise the doublet triplet splitting and make the model free from dangerous color triplets at scales below $M_{G U T}$. We choose $M_{5_{h_{u}}}=1$, to accommodate the Higgs $5_{h_{u}}$ and $M_{5_{h_{d}}}=0$ with $N_{8}=1$ so that we get the splitting of down quark in the Higgs fiveplet

$$
\Sigma_{5_{h_{d}}}:\left\{\begin{array}{l}
n_{(3,1)_{-1 / 3}}-n_{(\overline{3}, 1)_{1 / 3}}=M_{5_{5}}=0 \\
n_{(1,2)_{1 / 2}}-n_{(1,2)_{-1 / 2}}=M_{5_{5}}+N-N_{8}=-1
\end{array}\right.
$$


In order to satisfy the trace conditions we choose $M_{5_{6}}=-1, N_{7}=-1$ so that $\overline{5}^{(6)}$ has only a colour triplet component:

$$
\Sigma_{5^{(6)}}:\left\{\begin{array}{l}
n_{(3,1)_{-1 / 3}}-n_{(\overline{3}, 1)_{1 / 3}}=M_{5_{6}}=-1 \\
n_{(1,2)_{1 / 2}}-n_{(1,2)_{-1 / 2}}=M_{5_{6}}+N-N_{7}=0
\end{array}\right.
$$

In this simple example we have succeeded to disentangle the triplet from the up-Higgs curve at the price of generating however a new one in a different matter curve. Yet, this allows the possibility of realising the doublet-triplet splitting since we can generate a mass $M_{T}$ for the triplet by coupling it to an antitriplet via the appropriate superpotential term [18]. This way we obtain the corresponding Higgs doublets light.

However from Table 1 one may see that the matter on the $\Sigma_{10^{(2,3)}}$ curves will be affected by the $N_{7,8}$ flux. In particular the content of $10 / \overline{1} 0$-representations on $\Sigma_{10^{(2,3)}}$ is split by the choice of fluxes as follows

$$
\begin{gathered}
\Sigma_{10^{(2)}}: \begin{cases}n_{(3,2)_{1 / 6}}-n_{(\overline{3}, 2)_{-1 / 6}}=M_{10_{2}}=1 \\
n_{(\overline{3}, 1)_{-2 / 3}}-n_{(3,1)_{2 / 3}}=M_{10_{2}}-N_{7}=2 \\
n_{(1,1)_{1}}-n_{(1,1)_{-1}}=M_{10_{2}}+N_{7}=0\end{cases} \\
\Sigma_{10^{(3)}}: \begin{cases}n_{(3,2)_{1 / 6}}-n_{(\overline{3}, 2)_{-1 / 6}}=M_{10_{3}}=1 \\
n_{(\overline{3}, 1)_{-2 / 3}}-n_{(3,1)_{2 / 3}}=M_{10_{3}}-N_{8}=0 \\
n_{(1,1)_{1}}-n_{(1,1)_{-1}}=M_{10_{3}}+N_{8}=2 .\end{cases}
\end{gathered}
$$

We observe that in the presence of flux one $e^{c}=(1,1)_{1}$ state is 'displaced' from $\Sigma_{10^{(2)}}$ to the $\Sigma_{10^{(3)}}$ curve. A similar dislocation occurs for one $u^{c}=(\overline{3}, 1)_{-2 / 3}$ of $\Sigma_{10^{(3)}}$ which 'reappears' in $\Sigma_{10^{(2)}}$. We note that this fact implies a different structure for the up, down and charged lepton mass matrices. It can be checked that the particular distribution of the chiral matter on the specific matter curves can lead to interesting results with respect to the fermion mass structure and other phenomenological properties of the model [18]. For clarity, the final distribution of the MSSM spectrum along the available matter curves is summarized in Table 2 .

Here, we are interested for the renormalization group properties of the model. Using the above context we get the following relations

$$
\begin{array}{ll}
\sum_{i}\left|M_{5}^{i}\right|=5, & \sum_{i}\left|M_{5}^{i}+N_{5 Y}^{i}\right|=5 \\
\sum_{j}\left|M_{10}^{j}\right|=3, & \sum_{j}\left|M_{10}^{j}+N_{10 Y}^{j}\right|=3, \quad \sum_{j}\left|M_{10}^{j}-N_{10 Y}^{j}\right|=3
\end{array}
$$

which obviously respect the three constraints in (34) and at the same time

$$
-\sum_{i} M_{5}^{i}=\sum_{j} M_{10}^{j}=3
$$

which ensures three chiral families and the tracelessness condition (28). 


\begin{tabular}{|c|c|c|c|c|c|c|c|c|c|c|}
\hline & $\bar{M}$ & $N$ & $\bar{Q}$ & $\overline{u^{c}}$ & $e^{c}$ & & $\bar{M}$ & $N$ & $\overline{d^{c} / T}$ & $L / h_{u, d}$ \\
\hline $10^{(1)}\left(F_{3}\right)$ & 1 & 0 & 1 & 1 & 1 & $5^{(0)}\left(h_{u}, T\right)$ & 1 & 0 & 1 & 1 \\
\hline $10^{(2)}\left(F_{2,1}\right)$ & 1 & -1 & 1 & 2 & 0 & $5^{(1)}\left(\bar{f}_{2}\right)$ & -1 & 0 & -1 & -1 \\
\hline $10^{(3)}\left(F_{1,2}\right)$ & 1 & 1 & 1 & 0 & 2 & $5^{(2)}\left(\bar{f}_{3}\right)$ & -1 & 0 & -1 & -1 \\
\hline $10^{(4)}(-)$ & 0 & 0 & 0 & 0 & 0 & $5^{(3)}(-)$ & 0 & 0 & 0 & 0 \\
\hline & & & & & & $5^{(4)}\left(\bar{f}_{1}\right)$ & -1 & 0 & -1 & -1 \\
\hline & & & & & & $5^{(5)}\left(h_{d}\right)$ & 0 & -1 & 0 & -1 \\
\hline & & & & & & $5^{(6)}(\bar{T})$ & -1 & 1 & -1 & 0 \\
\hline
\end{tabular}

Table 2: The distribution of the chiral and Higgs matter content of the minimal model along the available curves, after the $U(1)_{Y}$ flux is turned on. The three families $F_{i}=$ $10_{i}, \bar{f}_{j}=\overline{5}_{j}$ are assigned on the curves as indicated. The Higgs doublets $h_{u, d}$ and $T / \bar{T}$ triplets descend from three different curves. (see also Table 1 and text.)

\section{The GUT scale and the flux thresholds}

In the previous section we have shown that the contribution to the $\beta$-functions $b_{a}^{x}$ of the chiral spectrum of the particular $S U(5)$ model is in the required ratio, i.e. proportional to the coefficients $k_{a}$.

We have already explained that in the present model we assume that the breaking of $S U(5)$ occurs when a flux is turned on along the $U(1)_{Y}$ component of the $S U(5)$ gauge symmetry. The rather interesting feature of the particular choice of fluxes leads to a minimal spectrum which consists of the MSSM content and only a color triplet pair below $M_{G U T}$. Furthermore, the appearance solely of (any number of) pairs of extra triplets -and no other additional matter- has the interesting property of not disturbing the scale where the unification is achieved. This fact is valid irrespectively of the scale that these triplets become massive. As a result, the nice properties of the minimal supersymmetric unification scenarios where the GUT scale is determined around $M_{G U T} \sim 10^{16} \mathrm{GeV}$ is retained. On the other hand, the value of the gauge coupling $g_{G U T}$ does depend on the number of triplets and the scale they become massive.

For convenience we start by recapitulating the basic analysis of the gauge coupling running in the presence of fluxes. In $F$-theory constructions the flux mechanism employed to break the GUT symmetry induces a splitting of the gauge couplings at the GUT scale [5,29]. The following relation is found at the unification scale [29]

$$
\frac{1}{a_{Y}\left(M_{G U T}\right)}=\frac{5}{3} \frac{1}{a_{1}\left(M_{G U T}\right)}=\frac{1}{a_{2}\left(M_{G U T}\right)}+\frac{2}{3} \frac{1}{a_{3}\left(M_{G U T}\right)}
$$

The running of the couplings is governed by the RGEs where contributions to the $\beta$ functions come from the MSSM spectrum and other possible remnants from the higher theory. In the case of the model under consideration we need to consider the simple case where in addition to low energy states only an additional triplet pair appears in the spectrum below the $S U(5)$-GUT breaking scale $M_{G U T}$. We assume that at some scale $M_{X}<M_{G U T}$ the extra triplet pair $T, \bar{T}$ decouples and only the MSSM spectrum remains massless for scales $\mu<M_{X}$. The low energy values of the gauge couplings are 
then given by the evolution equations

$$
\frac{1}{a_{a}\left(M_{Z}\right)}=\frac{1}{a_{a}\left(M_{G U T}\right)}+\frac{b_{a}^{x}}{2 \pi} \ln \frac{M_{G U T}}{M_{X}}+\frac{b_{a}}{2 \pi} \ln \frac{M_{X}}{M_{Z}}
$$

where $b_{a}^{x},\left(b_{a}\right)$ are the $\beta$-functions above (below) the scale $M_{X}$.

Using the GUT relation (41) one arrives at the following formula for the GUT scale

$$
M_{G U T}=e^{\frac{2 \pi}{\beta \mathcal{A}} \rho}\left(\frac{M_{X}}{M_{Z}}\right)^{1-\rho} M_{Z}
$$

where $\mathcal{A}$ is a function of the experimentally known low energy values of the SM gauge coupling constants

$$
\frac{1}{\mathcal{A}}=\frac{5}{3} \frac{1}{a_{1}\left(M_{Z}\right)}-\frac{1}{a_{2}\left(M_{Z}\right)}-\frac{2}{3} \frac{1}{a_{3}\left(M_{Z}\right)}
$$

and $\rho$ is the ratio

$$
\rho=\frac{\beta}{\beta_{x}}
$$

where $\beta, \beta_{x}$ are the $\beta$-functions combinations above and below the $M_{X}$ scale

$$
\begin{gathered}
\beta_{x}=b_{Y}^{x}-b_{2}^{x}-\frac{2}{3} b_{3}^{x} \\
\beta=b_{Y}-b_{2}-\frac{2}{3} b_{3}
\end{gathered}
$$

If the only additional states above $M_{X}$ are the color triplets $T=(3,1)_{1 / 3}, \bar{T}=$ $(\overline{3}, 1)_{-1 / 3}$, it can be easily checked that their total contribution to $\beta_{x}$ combination adds up to zero. This means that $\beta_{x}=\beta$ and $\rho=1$, thus $M_{G U T}$ in (43) becomes independent of the $M_{X}$ scale and in fact it is identified with the MSSM unification scale

$$
M_{U}=M_{G U T} \equiv e^{\frac{2 \pi}{\beta \mathcal{A}}} M_{Z} \approx 2.15 \times 10^{16} \mathrm{GeV}
$$

This result is only slightly modified when two loop corrections are taken into account [30]. The irrelevance of the triplets' decoupling scale $M_{X}$ holds for the one loop calculation and it is adequate for our purposes. With these preliminaries we are ready to discuss the computation of the Yukawa couplings.

\section{Wavefunction overlapping integrals}

In the class of models under consideration $U(1)$ symmetries are acting as family symmetries and fermion families are distributed over the curves in such a way so that fermion mass textures are rank one. Then the only tree level Yukawa couplings are those of the third generation and these are computed in terms of integrals over the overlapping wavefunctions at the triple intersection points

$$
\lambda_{i j}=M_{*}^{4} \int_{S} \psi_{i} \psi_{j} \phi d z_{1} \wedge d \bar{z}_{1} \wedge d z_{2} \wedge d \bar{z}_{2}
$$


Chiral matter is localized along the matter curves and therefore the value of the integral involving the trilinear (top and bottom) Yukawa couplings principally depends on the local shape of the corresponding wavefunctions. In addition, a crucial factor in the determination of the exact values is the global normalization of the wavefunctions which could in principle depend on the geometry of the compact manifold. In what follows, we will make use of the analysis of the previous section to argue that this dependence enters only through the torsion which according to Ray-Singer theorem is independent of the metric of the manifold.

To compute the integral we use the knowledge of the wavefunctions close to the intersection point. The solution of the zero-mode equations leads to a Gaussian profile of the wavefunctions $\psi$ which for localized solutions acquire the general form

$$
\psi \propto e^{-m^{2} \frac{\left|q_{1} z_{1}+q_{2} z_{2}\right|^{2}}{q}}
$$

where $m$ is a mass parameter related to some background Higgs vev and $q=\sqrt{q_{1}^{2}+q_{2}^{2}}$. We also need the normalization of the wavefunction which is determined by the integral:

$$
\mathcal{C}=M_{*}^{4} \int_{S}|\psi|^{2} d z \wedge d \bar{z}=\pi \frac{M_{*}^{4}}{m^{2} q} \mathcal{R}^{2}
$$

The factor $\pi \frac{M_{*}^{4}}{m^{2} q}$ is the result of the gaussian integration along the coordinate normal to the curve. The factor $\mathcal{R}^{2}$ is introduced to account for the integration along the coordinate parametrising the curve. We observe that in principle three different scales are introduced in the above normalization formula of the wavefunction, namely $M_{*}, m$ and $\mathcal{R}^{-1}$. Thus, although the solution of the equations of motion lead to a wavefunction which is peaked on the curve while falling off exponentially away from it, the final profile appears to comprise global information through the aforementioned mass parameters. We argue here that these apparently unrelated mass scales are connected by our previous analysis and the final wavefunction formula is solely characterised from an invariant quantity related to the torsion. Indeed, the scale $m$ that has been introduced in relation to the vacuum expectation value of a background scalar field in connection with the breaking of the enhanced gauge symmetry, is associated to the scale $M_{*} \approx M_{C}$ which appeared in (24). On the other hand, the parameter $\mathcal{R}$ 'measures' the integration along the matter curve inside the GUT surface $S$, thus clearly $\mathcal{R}^{-1}<M_{C}$ and from our previous analysis one naturally expects that $\mathcal{R}^{-1} \approx M_{K K} \approx M_{G U T}$. Although both scales are not known exactly, it is possible from our previous analysis on threshold corrections to obtain a scale independent normalization of the wavefunction. Indeed, following the reasoning presented in the above lines we can write (50) as follows

$$
\mathcal{C}=\frac{\pi}{q} \frac{M_{C}^{2}}{M_{G U T}^{2}}
$$

From the $M_{G U T}$ definition formula (23) we observe that the ratio of the two scales $M_{G U T} / M_{C}$ is independent of the geometry of the manifold and can be written as a 
simple exponential function of the torsion. Substituting (24) into (51) we find that the wavefunctions must be normalised with

$$
\frac{1}{\sqrt{C}}=\sqrt{\frac{q}{\pi}} e^{2 / 3\left(\mathcal{T}_{5 / 6}-\mathcal{T}_{0}\right)}
$$

Therefore, the normalization constant $C$ is found to be independent of the two scales $M_{G U T}, M_{C}$ of the theory.

Now, the computation of the integral for three generic wavefunctions of the form (48) with arbitrary charges $q_{i}, q_{i}^{\prime}, q_{i}^{\prime \prime}, i=1,2$ participating in the triple intersection the Yukawa couplings, gives

$$
\lambda=e^{2\left(\mathcal{T}_{5 / 6}-\mathcal{T}_{0}\right)} \frac{4 \sqrt{\pi}}{q+q^{\prime}+q^{\prime \prime}} \frac{\left(q q^{\prime} q^{\prime \prime}\right)^{3 / 2}}{\left(q_{1} q_{2}^{\prime}-q_{1}^{\prime} q_{2}\right)^{2}}
$$

where $q_{i}^{\prime \prime}=-q_{i}-q_{i}^{\prime}$ from charge conservation in the triple intersection, while $q=$ $\sqrt{q_{1}^{2}+q_{2}^{2}}$ and similarly for $q^{\prime}, q^{\prime \prime}[18$. We point out that the dependence on the value of gauge coupling $a_{G U T}$ in previous wavefunction normalizations has been replaced by the torsion. Previous normalizations where plagued by the smallness of the gauge coupling constant $a_{G U T} \sim \frac{1}{25}$ which led to rather small values of the top Yukawa coupling in disagreement with the data 7. The hope is that the replacement of this small factor with the exponential factor incorporating the analytic torsion might point to the correct answer.

In order to demonstrate the points discussed above, we proceed with the computation of the determinants specifying the value of the torsion in a simple case. As in ref [5] we take a line bundle $\mathcal{O}(n,-n)$ on a Hirzebruch surface $F_{0}=P^{1} \times P^{1}$. Taking into account that the Euler character on $P^{1}$ is given $\chi\left(P^{1}, \mathcal{O}(n)\right)=n+1$ the product formula of Ray-Singer torsion yields

$$
\mathcal{T}_{\mathcal{O}(n, m)}=(n+1) \mathcal{T}_{\mathcal{O}(m)}+(m+1) \mathcal{T}_{\mathcal{O}(n)}
$$

Since we want to eliminate the color triplets $(3,2)_{-5 / 6}+(\overline{3}, 2)_{5 / 6}$ we need to take $\chi\left(P^{1}, \mathcal{O}(n)\right)=n+1=0$, therefore for the line bundle $\mathcal{L}^{5 / 6}$ we take $n=-1$, so

$$
\mathcal{T}_{5 / 6}=\mathcal{T}_{\mathcal{O}(1,-1)}=2 \mathcal{T}_{\mathcal{O}(-1)}+(-1+1) \mathcal{T}_{\mathcal{O}(1)}=2 \mathcal{T}_{\mathcal{O}(-1)}
$$

For the trivial line bundle we take $n=0$, hence

$$
\mathcal{T}_{0}=\mathcal{T}_{\mathcal{O}(0,0)}=2 \mathcal{T}_{\mathcal{O}(0)}
$$

We now recall that a positive elliptic differential operator, as is the case of $\Delta_{k, R}$ with spectrum $\lambda_{n}$, is associated to the zeta functions [34,35]

$$
\zeta_{k}(s)=\sum_{\lambda_{n}} \frac{\gamma_{n}}{\lambda_{n}^{s}}
$$

\footnotetext{
${ }^{7}$ In previous normalizations, the appearance of the gauge coupling in the wavefunction normalization is due to the relation $a_{G U T}^{-1}=M_{*}^{4} \int \omega \wedge \omega \propto \mathcal{R}^{4} M_{*}^{4}$. However, in this approach, the result depends on the scaling of the metric and it is not clear whether the effective value $g_{G U T}$ or the coupling $g_{s}$ should be involved.
} 
where $\gamma_{n}$ is the degeneracy of $\lambda_{n}$. The determinant is defined by

$$
\log (\operatorname{Det} \Delta)=-\left.\frac{d \zeta_{k}(s)}{d s}\right|_{s=0}
$$

Therefore, the torsion is given $\mathcal{T}_{\mathcal{O}(k)}=-\frac{1}{2} \zeta_{k}^{\prime}(0)$ with

$$
\zeta_{k}^{\prime}(0)=4 \zeta_{R}^{\prime}(-1)-\frac{1}{2}(k+1)+\sum_{l=1}^{k+1}(2 l-|k+1|) \log l
$$

where $\zeta_{R}^{\prime}(-1) \approx-0.165421$. Then we get

$$
\mathcal{T}_{5 / 6}-\mathcal{T}_{0}=2\left(\mathcal{T}_{\mathcal{O}(-1)}-\mathcal{T}_{\mathcal{O}(0)}\right)=\zeta_{0}^{\prime}(0)-\zeta_{-1}^{\prime}(0)=-\frac{1}{2}
$$

For this particular choice of bundle, the Yukawa coupling then becomes

$$
\lambda=\frac{4 \sqrt{\pi} / e}{q+q^{\prime}+q^{\prime \prime}} \frac{\left(q q^{\prime} q^{\prime \prime}\right)^{3 / 2}}{\left(q_{1} q_{2}^{\prime}-q_{1}^{\prime} q_{2}\right)^{2}}
$$

\subsection{The top mass}

Let us now apply the above formula in the case of the model presented in the previous section. From Table 1 we see that a tree-level mass for the top quark is available from the gauge invariant Yukawa coupling

$$
\begin{array}{rrl}
W_{\text {tree }}=\lambda_{t} \mathbf{1 0}_{3} & \cdot \mathbf{1 0}_{3} & \cdot \mathbf{5}_{h_{u}} \\
t_{1} & t_{2} & -t_{1}-t_{2}
\end{array}
$$

where from the second line we see that the $U(1)$ invariance $\sum_{i} t_{i}=0$ is also satisfied. The charges in (53) for the top vertex for this model have been calculated in [18]

$$
\left\{q_{1}, q_{2}\right\}=\left\{\sqrt{\frac{3}{10}}, \frac{1}{\sqrt{2}}\right\},\left\{q_{1}^{\prime}, q_{2}^{\prime}\right\}=\left\{\sqrt{\frac{3}{10}},-\frac{1}{\sqrt{2}}\right\}
$$

The resulting top quark coupling is computed form (53) and is found to be $\lambda_{t} \approx$ 1.23. Similarly the bottom quark Yukawa coupling in the model is obtained form the superpotential term

$$
\begin{array}{rlcc}
W_{\text {tree }}=\lambda_{b} \mathbf{1 0}_{3} & \cdot \overline{\mathbf{5}}_{3} & \cdot \overline{\mathbf{5}}_{h_{d}} \\
t_{2} & t_{3}+t_{5} & t_{1}+t_{4}
\end{array}
$$

while repeating the above analysis one finds $\lambda_{b} \approx 1.17$. We thus observe that we are in the large $\tan \beta$ regime with a top coupling in the range $\lambda_{t} \geq 1$ which implies a physical mass around $186 \mathrm{GeV}$ which is within the experimental range [36].

In deriving the above numerical values we have made a specific simple choice of flat line bundle to compute the torsion which is involved in the top and bottom mass 


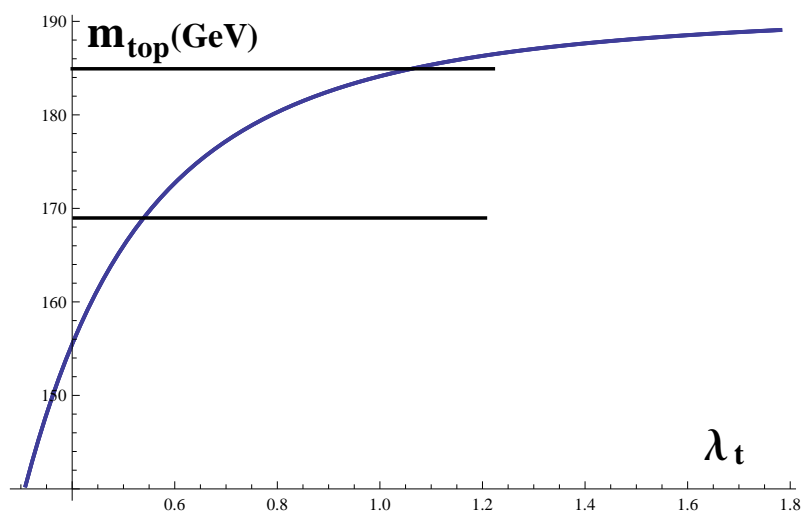

Figure 1: Plot of the top mass versus the value of the Yukawa coupling $\lambda_{t}\left(M_{G U T}\right)$, for $\tan \beta=(40-55)$. Horizontal lines indicate the experimental uncertainties of the top mass.

formula through the exponential factor appearing in (52). Of course, one may think of more involved cases of bundle structures however the above estimates are not expected to alter dramatically. Moreover, in a fully realistic model other parameters emerging from various thresholds in the renormalization group running etc may modify also the computation in obtaining the low energy masses. The final result will also depend on the specific choice of $\tan \beta$ which is fixed only by phenomenology and not by some theoretical principle. Thus, in our present computations we have the freedom to adjust its value to fit the low energy mass data. Furthermore, the reliability of our computations is corroborated from the rather interesting property of the top coupling, namely its fixed point behavior. This means that for a range of relatively large initial $\lambda_{t}$ values at GUT, the renormalized value of the top Yukawa approaches the same limiting value at the scale of $M_{W}$. It is anticipated that another choice of bundle would imply rea-

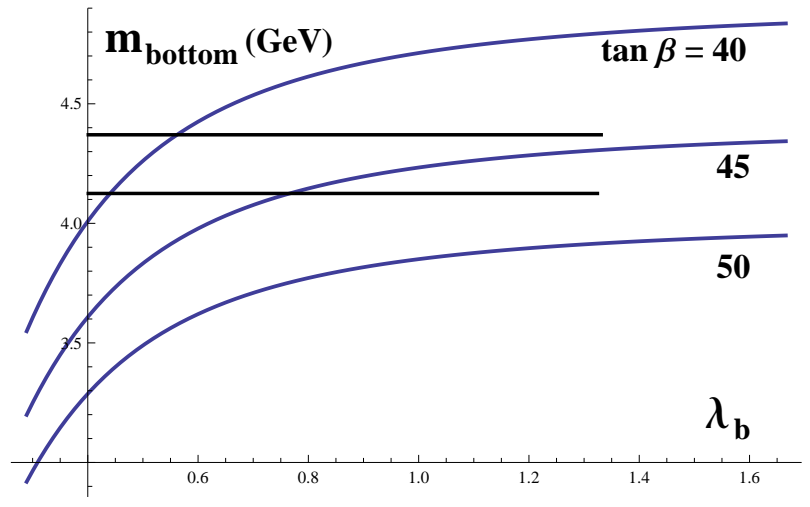

Figure 2: Plot of the bottom mass versus the value of the Yukawa coupling $\lambda_{b}\left(M_{G U T}\right)$ for three different values of $\tan \beta=(40,45,50)$. Horizontal lines indicate the experimental uncertainties on the bottom mass. 
sonable deviations from the numbers obtained in this particular example, so that they do not alter substantially the low energy predictions. In this sense, a fully realistic model could arise under an appropriate type of bundle structure. For completeness, we demonstrate the fixed point behavior by a one-loop renormalization group analysis using analytic formulae [39] of the top-bottom Yukawa coupled differential equations in the Appendix. To visualize this effect, using the derived formulae, in Fig.(1) and (2) we plot the low energy top and bottom masses as a function of the values of the corresponding Yukawa couplings at $M_{G U T}$. This is done for a range of large values of $\tan \beta \approx(40-55)$ as indicated in the plots. We can see clearly in the bottom mass plot the three curves corresponding to the values $\tan \beta=40,45,50$. Notice that in the case of the top mass plot, all $\tan \beta$ curves are squeezed to one because the top mass depends on $\sin (\beta) \lesssim \mathcal{O}(1)$. Horizontal lines specify the experimental uncertainties. It can be seen that top mass is found in a region where the Yukawa coupling is fairly close enough to the fixed point and small uncertainties in its computation are within the experimentally acceptable bounds.

\section{Conclusions}

In this work we have reconsidered the possibility of determining in a reliable way the mass scales and moduli parameters which enter in the computation of the tree level Yukawa couplings in a class of F-Theory GUT models. We consider in particular the $S U(5)$ GUT model generated by a seven brane wrapping a complex surface $S$ and embedded in the exceptional $E_{8}$ underlying theory. The $S U(5)$ GUT breaking is supposed to occur when a $U(1)_{Y}$ flux is turned on. In these models chiral fields are accommodated along the Riemann surfaces which constitute the intersections of the GUT brane with other 7 branes where the $S U(5)$ gauge symmetry is enhanced. Chiral matter fields lying on these 'curves' are charged under additional $U(1)$ 's which act as family symmetries. Under certain monodromy actions, Yukawa couplings can be formed at triple intersections of such curves and predict rank one fermion mass textures. The values of the Yukawa couplings are expressed in terms of integrals of overlapping wavefunctions of the matter fields participating in the triple intersection. The wavefunctions are found from the solution of the equations of motion and their normalization in principle appears to require knowledge of the mass scales of the theory and topological properties of the compact space and matter curves. We have made reasonable assumptions about the mass scales of the theory and used previous analysis of threshold effects from KK modes [5,27] to determine the GUT scale and the mass ratios which control the wavefunction normalization. Further, we have studied similar effects from a generic chiral sector of the F-theory model in the presence of $U(1)$ fluxes and have analysed the constraints imposed on the light spectrum of the effective field theory model. We have incorporated the above findings into the computation of generic trilinear top and bottom Yukawa couplings in the context of the $S U(5)$ model embedded in $E_{8}$ and found that the couplings depend only on the extra $U(1)$ charges carried by the fields at the intersection and a topological invariant quantity, namely the Ray-Singer 
torsion [28]. Furthermore, we have applied the method of our computation in a viable model obtained in the described F-theory context [18] with the minimal light spectrum which meets all the requirements imposed by our analysis. To compute the torsion, we have used [5] as example a simple case of flat line bundle on a Hirzebruch surface. We have performed the renormalization group analysis, taking into account the KK-mode effects discussed previously as well as flux thresholds to determine the low energy values of the gauge and Yukawa couplings. We have found a top and bottom mass which is in agreement with their experimentally determined values.

\section{Acknowledgement}

We would like to thank CERN theory group for kind hospitality during the last stage of this work. This work is partially supported by the European Research and Training Network (RTN) grant "Unification in the LHC era"(PITN-GA-2009-237920). 


\section{Appendix}

In subsection 6.1 we applied our method for the computation of the top and bottom Yukawa couplings which for a specific choice of line bundle they where found to obtain $\mathcal{O}(1)$ and comparable values (large $\tan \beta$ regime) at the GUT scale. The top and bottom masses are given from their renormalised values at scales $\sim M_{W}$, and are obtained from a solution of two coupled differential equations (DEs) (we assume for simplicity small tau Yukawa coupling). For comparable initial $\lambda_{t, b}$ values the DEs cannot be disentangled, thus in order to give a reliable calculation of their low energy values, we need to solve the above DEs and plot the values of the top and bottom masses for reasonable ranges of $\lambda_{b, t}$ initial values. To this end, in this Appendix we obtain analytic formulae by extending the analytic solution [37] of the system of the 1-loop RGE for the top and bottom Yukawa couplings $h_{t}$ and $h_{b}$. Defining $\alpha_{t}=\lambda_{t}^{2} /(4 \pi)$ and $\alpha_{b}=\lambda_{b}^{2} /(4 \pi)$, the RGEs are

$$
\begin{aligned}
\frac{d \alpha_{t}}{d t} & =\frac{1}{2 \pi}\left(6 \alpha_{t}+\alpha_{b}-G_{Q}\right) \alpha_{t} \\
\frac{d \alpha_{b}}{d t} & =\frac{1}{2 \pi}\left(\alpha_{t}+6 \alpha_{b}-G_{D}\right) \alpha_{b}
\end{aligned}
$$

with $G_{I}=c_{1}^{I} \alpha_{1}+c_{2}^{I} \alpha_{2}+c_{3}^{I} \alpha_{3}, I=Q, D$, and the constants $c$ are given by $(S U(5)$ normalization)

$$
\left\{c_{1}^{Q}, c_{2}^{Q}, c_{3}^{Q}\right\}=\left\{\frac{16}{3}, 3, \frac{13}{15}\right\}, \quad \text { and } \quad\left\{c_{1}^{D}, c_{2}^{D}, c_{3}^{D}\right\}=\left\{\frac{16}{3}, 3, \frac{7}{15}\right\}
$$

Ignoring the small difference in the $U(1)$ factor of the constants $c$, we shall make the transformations

$$
\alpha_{t}=\gamma_{Q}^{2} x, \quad \text { and } \quad \alpha_{b}=\gamma_{D}^{2} y \sim \gamma_{Q}^{2} y
$$

where

$$
\gamma_{Q}^{2}=\exp \left[-\frac{1}{2 \pi} \int_{t_{0}}^{t} G_{Q}\left(t^{\prime}\right) d t^{\prime}\right]
$$

The system (55) becomes

$$
\begin{aligned}
& \frac{d x}{d t}=\frac{1}{2 \pi}(6 x+y) \gamma_{Q}^{2} x \\
& \frac{d y}{d t}=\frac{1}{2 \pi}(x+6 y) \gamma_{Q}^{2} y
\end{aligned}
$$

Simple manipulations give

$$
\begin{aligned}
\frac{d \ln (x-y)}{d t} & =\frac{\gamma_{Q}^{2}}{2 \pi} 6(x+y) \\
\frac{d \ln (x y)}{d t} & =\frac{\gamma_{Q}^{2}}{2 \pi} 7(x+y)
\end{aligned}
$$

Therefore

$$
7 \frac{d \ln (x-y)}{d t}=6 \frac{d \ln (x y)}{d t}, \quad \text { or } \quad\left(\frac{x y}{x_{0} y_{0}}\right)^{6}=\left(\frac{x-y}{x_{0}-y_{0}}\right)^{7}
$$


where $x_{0}$ and $y_{0}$ are initial conditions at the scale $t_{0}$. Observe that, since $\gamma_{Q}^{2}\left(t_{0}\right)=1$, we have that $x_{0}=\alpha_{t}\left(t_{0}\right)$ and $y_{0}=\alpha_{b}\left(t_{0}\right)$. Using (59) we can write

$$
(x+y)^{2}=(x-y)^{2}+4 x y=(x-y)^{2}+4 \frac{x_{0} y_{0}}{\left(x_{0}-y_{0}\right)^{7 / 6}}(x-y)^{7 / 6}
$$

and the first of the equation in (58) reads

$$
\frac{d(x-y)}{d t}=\frac{\gamma_{Q}^{2}}{2 \pi} 6(x-y) \sqrt{(x-y)^{2}+4 \frac{x_{0} y_{0}}{\left(x_{0}-y_{0}\right)^{7 / 6}}(x-y)^{7 / 6}}
$$

Defining a further new transformation

$$
u=k_{0} / \omega^{5 / 6}=k_{0} /(x-y)^{5 / 6}, \quad \text { where } \quad k_{0}=4 \frac{x_{0} y_{0}}{\left(x_{0}-y_{0}\right)^{7 / 6}}
$$

we get

$$
\begin{aligned}
\frac{d u}{d t} & =\left(-\frac{5}{6}\right) \frac{k_{0}}{\omega^{11 / 6}} \frac{d \omega}{d t}=-\frac{5}{2 \pi} \frac{k_{0}}{\omega^{11 / 6}} \gamma_{Q}^{2} \omega \sqrt{\omega^{2}+k_{0} \omega^{7 / 6}} \\
& =-\frac{5}{2 \pi} \gamma_{Q}^{2} k_{0} \frac{u}{k_{0}} \sqrt{\left(\frac{k_{0}}{u}\right)^{12 / 5}+k_{0}\left(\frac{k_{0}}{u}\right)^{7 / 5}} \\
& =-\frac{5}{2 \pi} \gamma_{Q}^{2} k_{0}^{6 / 5} u \frac{\sqrt{1+u}}{u^{6 / 5}} \\
& =-\frac{5}{2 \pi} \gamma_{Q}^{2} k_{0}^{6 / 5} \frac{\sqrt{1+u}}{u^{1 / 5}}
\end{aligned}
$$

Therefore

$$
\frac{u^{1 / 5}}{\sqrt{1+u}} d u=-\frac{5}{2 \pi} k_{0}^{6 / 5} \gamma_{Q}^{2} d t
$$

Integrating the left-hand side we get

$$
\int \frac{u^{1 / 5}}{\sqrt{1+u}} d u=\frac{10}{7} u^{1 / 5}\left[\sqrt{1+u}-{ }_{2} \mathcal{F}_{1}\left(\frac{1}{5}, \frac{1}{2}, \frac{6}{5},-u\right)\right]
$$

where ${ }_{2} \mathcal{F}_{1}$ is the hypergeometric function. Let us work on the right-hand side of (61)

$$
\gamma_{Q}^{2}=\exp \left[-\frac{1}{2 \pi} \int_{t_{0}}^{t} G_{Q}\left(t^{\prime}\right) d t^{\prime}\right]=\exp \left[-\frac{1}{2 \pi} \int_{t_{0}}^{t}\left(c_{1}^{Q} \alpha_{1}\left(t^{\prime}\right)+c_{2}^{Q} \alpha_{2}\left(t^{\prime}\right)+c_{3}^{Q} \alpha_{3}\left(t^{\prime}\right)\right) d t^{\prime}\right]
$$

Since

$$
\int_{t_{0}}^{t} \alpha_{i}\left(t^{\prime}\right) d t^{\prime}=\int_{t_{0}}^{t} \frac{\alpha_{i 0} d t^{\prime}}{1-\frac{b_{i} \alpha_{i 0}}{2 \pi}\left(t^{\prime}-t_{0}\right)}=-\frac{2 \pi}{b_{i}} \ln \left[1-\frac{b_{i} \alpha_{i 0}}{2 \pi}\left(t-t_{0}\right)\right]
$$

we get

$$
\gamma_{Q}^{2}=\prod_{i=1}^{3}\left[1-\frac{b_{i} \alpha_{i 0}}{2 \pi}\left(t-t_{0}\right)\right]^{c_{i}^{Q} / b_{i}}
$$


Define for convenience the new constants (we suppress hereafter the superscript $Q$ )

$$
b_{i} \alpha_{i 0} /(2 \pi)=B_{i}, \quad \text { and } \quad c_{i} / b_{i}=e_{i}
$$

Integrating $\gamma_{Q}^{2}$ we get

$$
\begin{aligned}
& \int \gamma_{Q}^{2} d t=\frac{1}{B_{1}\left(1+e_{1}\right)}\left[-1+B_{1}\left(t-t_{0}\right)\right]\left[1-B_{1}\left(t-t_{0}\right)\right]^{e_{1}}\left[1-B_{2}\left(t-t_{0}\right)\right]^{e_{2}} \times \\
& {\left[\frac{B_{1}}{B_{1}-B_{2}}\left(1-B_{2}\left(t-t_{0}\right)\right)\right]^{-e_{2}}\left[1-B_{3}\left(t-t_{0}\right)\right]^{e_{3}}\left[\frac{B_{1}}{B_{1}-B_{3}}\left(1-B_{3}\left(t-t_{0}\right)\right)\right]^{-e_{3}} \times} \\
& { }_{A} \mathcal{F}\left(1+e_{1},-e_{2},-e_{3}, 2+e_{1}, \frac{B_{2}\left(-1+B_{1}\left(t-t_{0}\right)\right)}{B_{1}-B_{2}}, \frac{B_{3}\left(-1+B_{1}\left(t-t_{0}\right)\right)}{B_{1}-B_{3}}\right)
\end{aligned}
$$

where ${ }_{A} \mathcal{F}$ is the Appell hypergeometric function of two variables [38. Simplifying the relation and using $1-B_{1}\left(t-t_{0}\right)=\alpha_{10} / \alpha_{1}(t)$, we get

$$
\begin{gathered}
\int \gamma_{Q}^{2} d t=\frac{1}{B_{1}\left(1+e_{1}\right)}\left[-\left(\frac{\alpha_{10}}{\alpha_{1}(t)}\right)^{e_{1}+1}\right]\left[\frac{B_{1}-B_{2}}{B_{1}}\right]^{e_{2}}\left[\frac{B_{1}-B_{3}}{B_{1}}\right]^{e_{3}} \times \\
{ }_{A} \mathcal{F}\left(1+e_{1},-e_{2},-e_{3}, 2+e_{1}, \frac{B_{2}}{B_{2}-B_{1}}\left(\frac{\alpha_{10}}{\alpha_{1}(t)}\right), \frac{B_{3}}{B_{3}-B_{1}}\left(\frac{\alpha_{10}}{\alpha_{1}(t)}\right)\right)
\end{gathered}
$$

Therefore the differential equation (61) gives

$$
\begin{aligned}
& \frac{10}{7} u^{1 / 5}\left[\sqrt{1+u}-{ }_{2} \mathcal{F}_{1}\left(\frac{1}{5}, \frac{1}{2}, \frac{6}{5},-u\right)\right]= \\
& -\frac{5}{2 \pi} k_{0}^{6 / 5} \frac{1}{B_{1}\left(1+e_{1}\right)}\left[-\left(\frac{\alpha_{10}}{\alpha_{1}(t)}\right)^{e_{1}+1}\right]\left[\frac{B_{1}-B_{2}}{B_{1}}\right]^{e_{2}}\left[\frac{B_{1}-B_{3}}{B_{1}}\right]^{e_{3}} \times \\
& { }_{A} \mathcal{F}\left(1+e_{1},-e_{2},-e_{3}, 2+e_{1}, \frac{B_{2}}{B_{2}-B_{1}}\left(\frac{\alpha_{10}}{\alpha_{1}(t)}\right), \frac{B_{3}}{B_{3}-B_{1}}\left(\frac{\alpha_{10}}{\alpha_{1}(t)}\right)\right)+\text { constant }
\end{aligned}
$$

and simplifying

$$
\begin{aligned}
& u^{1 / 5}\left[\sqrt{1+u}-{ }_{2} \mathcal{F}_{1}\left(\frac{1}{5}, \frac{1}{2}, \frac{6}{5},-u\right)\right]= \\
& \frac{7}{4 \pi} k_{0}^{6 / 5} \frac{1}{B_{1}\left(1+e_{1}\right)}\left[\left(\frac{\alpha_{10}}{\alpha_{1}(t)}\right)^{e_{1}+1}\right]\left[\frac{B_{1}-B_{2}}{B_{1}}\right]^{e_{2}}\left[\frac{B_{1}-B_{3}}{B_{1}}\right]^{e_{3}} \times \\
& { }_{A} \mathcal{F}\left(1+e_{1},-e_{2},-e_{3}, 2+e_{1}, \frac{B_{2}}{B_{2}-B_{1}}\left(\frac{\alpha_{10}}{\alpha_{1}(t)}\right), \frac{B_{3}}{B_{3}-B_{1}}\left(\frac{\alpha_{10}}{\alpha_{1}(t)}\right)\right)+\text { constant }
\end{aligned}
$$

Let us see now how $x$ and $y$ are related with $\omega$ which, from (60) is given in terms of $u$. Since (see (59))

$$
x-y=x+(-y)=\omega, \quad \text { and } \quad(-y) \cdot x=-x_{0} y_{0}\left(\frac{\omega}{\omega_{0}}\right)^{7 / 6}
$$


we get (using (60) for the second equality)

$$
\begin{aligned}
& x=\frac{\omega}{2}\left(1+\sqrt{1+k_{0} \omega^{-5 / 6}}\right)=\frac{1}{2}\left(\frac{k_{0}}{u}\right)^{6 / 5}(1+\sqrt{1+u}) \\
& y=\frac{\omega}{2}\left(-1+\sqrt{1+k_{0} \omega^{-5 / 6}}\right)=\frac{1}{2}\left(\frac{k_{0}}{u}\right)^{6 / 5}(-1+\sqrt{1+u})
\end{aligned}
$$

From (56) we get

$$
\begin{aligned}
& \alpha_{t}=\gamma_{Q}^{2} x=\prod_{i=1}^{3}\left[1-\frac{b_{i} \alpha_{i 0}}{2 \pi}\left(t-t_{0}\right)\right]^{c_{i}^{Q} / b_{i}} \frac{1}{2}\left(\frac{k_{0}}{u}\right)^{6 / 5}(1+\sqrt{1+u}) \\
& \alpha_{b}=\gamma_{D}^{2} y=\prod_{i=1}^{3}\left[1-\frac{b_{i} \alpha_{i 0}}{2 \pi}\left(t-t_{0}\right)\right]^{c_{i}^{D} / b_{i}} \frac{1}{2}\left(\frac{k_{0}}{u}\right)^{6 / 5}(-1+\sqrt{1+u})
\end{aligned}
$$

These formulae incorporate the RG running effects and encompass all the information for the initial values of the gauge and Yukawa couplings at the GUT scale. We may substitute the top and bottom initial values and use them [39] to derive our results. 


\section{References}

[1] R. Donagi and M. Wijnholt, "Model Building with F-Theory", arXiv:0802.2969 [hep-th].

[2] C. Beasley, J. J. Heckman and C. Vafa, "GUTs and Exceptional Branes in F-theory - I,” JHEP 0901 (2009) 058 arXiv:0802.3391 [hep-th]].

[3] C. Beasley, J. J. Heckman and C. Vafa, "GUTs and Exceptional Branes in Ftheory - II: Experimental Predictions," JHEP 0901 (2009) 059 arXiv:0806.0102 [hep-th]].

[4] H. Hayashi, R. Tatar, Y. Toda, T. Watari and M. Yamazaki, "New Aspects of Heterotic-F Theory Duality," Nucl. Phys. B 806 (2009) 224 [arXiv:0805.1057[hepth]].

[5] R. Donagi and M. Wijnholt, "Breaking GUT Groups in F-Theory," arXiv:0808.2223 [hep-th].

[6] J. J. Heckman, A. Tavanfar, C. Vafa, "The Point of E(8) in F-theory GUTs," JHEP 1008, 040 (2010). [arXiv:0906.0581 [hep-th]]

[7] J. Marsano, N. Saulina and S. Schafer-Nameki, "Compact F-theory GUTs with $U(1)_{P} Q$," JHEP 1004 (2010) 095 [arXiv:0912.0272 [hep-th]].

[8] R. Blumenhagen, T. W. Grimm, B. Jurke and T. Weigand, "F-theory uplifts and GUTs," JHEP 0909 (2009) 053 arXiv:0906.0013 [hep-th]].

[9] T. W. Grimm, "The N=1 effective action of F-theory compactifications," Nucl. Phys. B 845 (2011) 48 [arXiv:1008.4133 [hep-th]].

[10] T. Weigand, "Lectures on F-theory compactifications and model building," Class. Quant. Grav. 27 (2010) 214004 arXiv:1009.3497 [hep-th]].

[11] J. J. Heckman and C. Vafa, "Flavor Hierarchy From F-theory," Nucl. Phys. B 837 (2010) 137 [arXiv:0811.2417 [hep-th]].

[12] S. Cecotti, M. C. N. Cheng, J. J. Heckman and C. Vafa, "Yukawa Couplings in F-theory and Non-Commutative Geometry," arXiv:0910.0477 [hep-th].

[13] J. Marsano, N. Saulina and S. Schafer-Nameki, "Monodromies, Fluxes, and Compact Three-Generation F-theory GUTs," JHEP 0908 (2009) 046 arXiv:0906.4672 [hep-th]].

[14] J. P. Conlon and E. Palti, "Aspects of Flavour and Supersymmetry in F-theory GUTs," JHEP 1001 (2010) 029 [arXiv:0910.2413 [hep-th]].

[15] E. Dudas and E. Palti, "Froggatt-Nielsen models from E8 in F-theory GUTs," JHEP 1001 (2010) 127 arXiv:0912.0853 [hep-th]]. 
[16] S. F. King, G. K. Leontaris and G. G. Ross, "Family symmetries in F-theory GUTs," Nucl. Phys. B 838 (2010) 119 [arXiv:1005.1025 [hep-ph]].

[17] E. Dudas and E. Palti, "On hypercharge flux and exotics in F-theory GUTs," arXiv:1007.1297 [hep-ph].

[18] G. K. Leontaris, G. G. Ross, "Yukawa couplings and fermion mass structure in F-theory GUTs," arXiv:1009.6000 [hep-th]].

( To appear in JHEP.)

[19] H. Hayashi, T. Kawano, Y. Tsuchiya and T. Watari, "Flavor Structure in F-theory Compactifications," JHEP 1008 (2010) 036 [arXiv:0910.2762 [hep-th]].

[20] T. W. Grimm and T. Weigand, "On Abelian Gauge Symmetries and Proton Decay in Global F-theory GUTs," Phys. Rev. D 82 (2010) 086009 arXiv:1006.0226 [hepth]].

[21] C. Ludeling, H. P. Nilles, C. C. Stephan, "The Potential Fate of Local Model Building," arXiv:1101.3346 [hep-th]].

[22] T. Li, D. V. Nanopoulos and J. W. Walker, "Fast proton decay," Phys. Lett. B 693 (2010) 580.

[23] E. Kuflik and J. Marsano, "Comments on Flipped SU(5) (and F-theory)," arXiv:1009.2510 [hep-ph].

[24] H. Hayashi, T. Kawano, R. Tatar and T. Watari, "Codimension-3 Singularities and Yukawa Couplings in F-theory," Nucl. Phys. B 823 (2009) 47 arXiv:0901.4941 [hep-th]].

[25] A. Font and L. E. Ibanez, "Matter wave functions and Yukawa couplings in Ftheory Grand Unification," JHEP 0909 (2009) 036 [arXiv:0907.4895 [hep-th]].

[26] F. Marchesano, P. McGuirk and G. Shiu, "Chiral matter wavefunctions in warped compactifications," arXiv:1012.2759 [hep-th].

[27] T. Friedmann and E. Witten, "Unification scale, proton decay, and manifolds of G(2) holonomy," Adv. Theor. Math. Phys. 7 (2003) 577 arXiv:hep-th/0211269.

[28] D. B. Ray and I. M. Singer, "Analytic Torsion For Complex Manifolds," Annals Math. 98 (1973) 154.

[29] R. Blumenhagen, "Gauge Coupling Unification In F-Theory Grand Unified Theories," Phys. Rev. Lett. 102 (2009) 071601 arXiv:0812.0248 [hep-th]].

[30] G. K. Leontaris and N. D. Tracas, "Gauge coupling flux thresholds, exotic matter and the unification scale in F-SU(5) GUT," Eur. Phys. J. C 67 (2010) 489 arXiv:0912.1557 [hep-ph]]. 
[31] J.P. Conlon and E. Palti, "On Gauge Threshold Corrections for Local IB/F-theory GUTs" Phys. Rev. D 80 (2009) 106004. arXiv:0907.1362 [hep-th]

[32] J. Jiang, T. Li, D. V. Nanopoulos and D. Xie, "Flipped $S U(5) X U(1)_{X}$ Models from F-Theory," Nucl. Phys. B 830 (2010) 195 [arXiv:0905.3394 [hep-th]].

[33] T. Li, D. V. Nanopoulos and J. W. Walker, "Elements of F-ast Proton Decay," Nucl. Phys. B 846 (2011) 43 [arXiv:1003.2570 [hep-ph]].

[34] D. B. Ray and I. M. Singer, "R-torsion and the Laplacian in Riemannian Manifolds," Advances in Mathematics 7 (1971) 145-210.

[35] C. Nash and D. J. O'Connor, "Determinants of Laplacians, the Ray-Singer torsion on lens spaces and the Riemann zeta function," J. Math. Phys. 36 (1995) 1462 [Erratum-ibid. 36 (1995) 4549] arXiv:hep-th/9212022].

[36] T. Aaltonen et al. [The CDF Collaboration], "Measurement of the Top Quark Mass in the Lepton+Jets Channel Using the Lepton Transverse Momentum," arXiv:1101.4926 [hep-ex].

[37] E. G. Floratos and G. K. Leontaris, "Large top and bottom Yukawa couplings in minimal supersymmetry," Phys. Lett. B 336 (1994) 194 arXiv:hep-ph/9407229].

[38] Appell, P., "Sur les fonctions hypergiomitriques de plusieurs variables." In Mimoir. Sci. Math. Paris: Gauthier-Villars, 1925. http://mathworld.wolfram.com/AppellHypergeometricFunction.html

[39] E. G. Floratos, G. K. Leontaris and S. Lola, "MSSM phenomenology in the large tan beta regime," Phys. Lett. B 365 (1996) 149 arXiv:hep-ph/9507402. 